\title{
Scale Invariance of Principle of Equivalent Utility under Cumulative Prospect Theory
}

\author{
J. Chudziak ${ }^{a *}$, M. HAlicki ${ }^{b}$, S. WÓJCiK ${ }^{c}$ \\ ${ }^{a}$ Faculty of Mathematics and Natural Sciences, University of Rzeszów, \\ Prof. St. Pigonia 1, 35-310 Rzeszów, Poland \\ ${ }^{b}$ Department of Regional Politics and Food Economy, University of Rzeszów, \\ Ćwiklińskiej 2, 35-601 Rzeszów, Poland \\ ${ }^{c}$ Statistical Office in Rzeszów, Jana III Sobieskiego 10, 35-959 Rzeszów, Poland
}

\begin{abstract}
In 1984 A. Reich proved that under Expected Utility Theory, a scale invariance of the Principle of Equivalent Utility just for two particular values of parameters implies its scale invariance. In this paper, we extend this result onto the Principle of Equivalent Utility under Cumulative Prospect Theory.

DOI: 10.12693/APhysPolA.127.A-29
\end{abstract}

PACS: 89.65.Gh

\section{Introduction}

Let us consider an insurance company having the initial wealth $w$ and a utility function $u$. The company covers a risk treated as a non-negative random variable. Roughly speaking, a premium principle is a rule for assigning a premium to an insured risk. One of the frequently applied methods of pricing insurance contracts is the Principle of Equivalent Utility. Under the Expected Utility Theory a premium principle $H(X)$ for risk $X$ is a solution of the equation

$$
u(w)=E[u(w+H(X)-X)] .
$$

A solution $H(X)$ of (1) in the case $w=0$ is called the zero-utility principle. Equation (1) has the following interpretation: the utility of the initial wealth is equal to the expected utility of the surplus that results when a risk $X$ is insured. A value of $H(X)$ is such that the insurer is indifferent between not accepting and accepting the insurance risk. Thus, this premium is called also the indifference price of the insurer.

It is known that the classical model of decision under risk, the Expected Utility model, has been violated by observed behaviors (e.g. the Allais paradox). Several authors weakened the independence axiom and presented the version of the Expected Utility models which explain the paradoxes. One of them is the Rank Dependent Utility model [1, 2]. Under that model the preference relation can be characterized by a Choquet integral. Let us recall that the Choquet integral has been introduced by Gustave Choquet in 1954 as a tool for some problems in statistical mechanics and potential theory.

Given a distortion function $g:[0,1] \rightarrow[0,1]$, that is a non-decreasing function with $g(0)=0$ and $g(1)=1$, and a random variable $X$, the Choquet integral related to $g$ is defined in the following way

$$
E_{g}(X)=\int_{-\infty}^{0}(g(P(X>t))-1) \mathrm{d} t+\int_{0}^{\infty} g(P(X>t)) \mathrm{d} t .
$$

\footnotetext{
*corresponding author; e-mail: chudziak@ur.edu.pl
}

In particular, if $x$ and $y$ are real numbers with $x<y$, $p \in[0,1]$ and $X$ is such that $P(X=x)=1-p$ and $P(X=y)=p$, then

$$
E_{g}(X)=(1-g(p)) x+g(p) y .
$$

Tversky and Kahneman [3], using the concept of the rank-dependent utility model, created the Cumulative Prospect Theory. They assumed that probabilities of gains and losses are distorted in a different way. Moreover, they introduced the generalized Choquet integral

$$
E_{g h}(X)=E_{g}(\max \{X, 0\})-E_{h}(\max \{-X, 0\})
$$

related to the distortion functions $g$ (for gains) and $h$ (for losses) and applied it to describe the mathematical foundations of the Cumulative Prospect Theory. The generalized Choquet integral has several interesting properties. In particular, it is monotonic and $E_{g h} c=c$ for $c \in \mathbb{R}$ (cf. [4]).

The Principle of Equivalent Utility under the Rank Dependent Utility model has been considered by Heilpern [5]. In this case equation (1) becomes

$$
u(w)=E_{g}[u(w+H(X)-X)] .
$$

In a recent paper Kałuszka and Krzeszowiec [4] introduced a modification of the Principle of Equivalent Utility adjusted to the Cumulative Prospect Theory. This approach leads to the equation

$$
u(w)=E_{g h}[u(w+H(X)-X)] .
$$

In the paper of Kałuszka and Krzeszowiec [4] several properties of the premium principle have been considered. One of them is a scale invariance, known also as a positive homogeneity. Let us recall that, for any $a>0$, a premium principle is said to be a-scale invariant provided $H(a X)=a H(X)$ for all feasible risks $X$. A premium principle is said to be scale invariant if it is $a$-scale invariant for every $a>0$.

An important justification for a scale invariance follows from the fact that a premium principle could be priced in two (or more) different currencies. As it has been noted in the paper of Reich [6], under Expected Utility Theory, a scale invariance of the Principle of Equivalent Utility just for two particular values of parameter $a$ implies its 
scale invariance. In this paper, we extend this result onto the Principle of Equivalent Utility under Cumulative Prospect Theory.

\section{Results}

In what follows, given an $x>0$ and a $p \in[0,1]$, by $(x, p)$ we denote the random variable $X$ such that $P(X=$ $0)=1-p$ and $P(X=x)=p$. Furthermore, we set $\mathcal{X}_{2}:=\{(x, p) \mid x>0, p \in[0,1]\}$.

Theorem 1. Assume that $u: \mathbb{R} \rightarrow \mathbb{R}$ is a strictly increasing and continuous utility function with $u(0)=0$ and $g, h$ are continuous distortion functions for gains and losses, respectively, such that

$$
g(1-p) h(p)>0 \text { for } p \in(0,1) .
$$

Let $a_{1}, a_{2} \in(0, \infty) \backslash\{1\}$ be such that $\frac{\ln a_{1}}{\ln a_{2}}$ is irrational. Assume that (4) holds for $w=0$ and $H$ is $a_{i}$-scale invariant for $i=1,2$ and every $X \in \mathcal{X}_{2}$. Then there exist $\alpha, \beta, d>0$ such that

$$
u(x)= \begin{cases}\alpha x^{d} & \text { for } x \geq 0, \\ -\beta(-x)^{d} & \text { for } x<0 .\end{cases}
$$

Conversely, if (5) holds and $u$ is of the form (6) with some $\alpha, \beta, d>0$, then $H$ is scale invariant.

Theorem 2. Assume that $u: \mathbb{R} \rightarrow \mathbb{R}$ is a strictly increasing and continuous utility function with $u(0)=0$ and $g, h$ are continuous distortion functions for gains and losses, respectively, such that (5) is satisfied. Let $a_{1}, a_{2} \in(0, \infty) \backslash\{1\}$ be such that $\frac{\ln a_{1}}{\ln a_{2}}$ is irrational. Assume that (4) holds for $w=0$ and some $w_{0}>0$; and $H$ is $a_{i}$-scale invariant for $i=1,2$ and every $X \in \mathcal{X}_{2}$. Then

$$
h(p)=1-g(1-p) \text { for } p \in(0,1)
$$

and there exist an $\alpha>0$ such that

$$
u(x)=\alpha x \text { for } x \in \mathbb{R} \text {. }
$$

\section{Proofs}

We begin this section with a result which will play an important role in the proofs of Theorems 1 and 2 .

Lemma. Assume that $a_{1}, a_{2} \in(0, \infty) \backslash\{1\}$ are such that $\frac{\ln a_{1}}{\ln a_{2}}$ is irrational, $c \in \mathbb{R} \backslash\{0\}$ and $f: \mathbb{R} \rightarrow \mathbb{R}$ is a strictly monotonic continuous function satisfying equation

$$
f\left(a_{i} x\right)=c f\left(a_{i}\right) f(x) \text { for } x \in(0, \infty), i \in\{1,2\}
$$

and such that $c f(x)>0$ for $x \in(0, \infty)$. Then there is a $d \in(0, \infty)$ such that

$$
f(x)=\frac{1}{c} x^{d} \text { for } x \in(0, \infty) .
$$

Proof. Let $F: \mathbb{R} \rightarrow \mathbb{R}$ be given by

$$
F(x)=\ln c f\left(e^{x}\right) \text { for } x \in \mathbb{R} \text {. }
$$

Then, in view of (9), we obtain

$$
\begin{aligned}
& F\left(\ln x+\ln a_{i}\right)=F\left(\ln a_{i} x\right)=\ln c f\left(a_{i} x\right)= \\
& \ln c^{2} f\left(a_{i}\right) f(x)=\ln c f(x)+\ln c f\left(a_{i}\right)= \\
& F(\ln x)+F\left(\ln a_{i}\right) \text { for } x \in(0, \infty), i \in\{1,2\} .
\end{aligned}
$$

Hence

$$
F\left(y+\ln a_{i}\right)=F(y)+F\left(\ln a_{i}\right) \text { for } y \in \mathbb{R}, i \in\{1,2\}
$$

and so, according to Lemma 18.5.1 in Kuczma [7],

$$
\begin{aligned}
& F(y+z)=F(y)+F(z) \text { for } y \in \mathbb{R}, \\
& \quad z \in G\left(\ln a_{1}, \ln a_{2}\right),
\end{aligned}
$$

where $G\left(\ln a_{1}, \ln a_{2}\right)$ denotes the subgroup of the additive group of real numbers generated by the set $\left\{\ln a_{1}, \ln a_{2}\right\}$. Furthermore, since $\frac{\ln a_{1}}{\ln a_{2}}$ is irrational, $G\left(\ln a_{1}, \ln a_{2}\right)$ is a dense subset of $\mathbb{R}$. Note also that as $f$ is strictly monotonic and continuous, so is $F$. Therefore from (12) it follows that $F$ is a nonconstant continuous additive function, that is

$$
F(y+z)=F(y)+F(z) \text { for } y, z \in \mathbb{R} .
$$

Hence, applying Theorem 5.5.2 in Kuczma [7], we obtain that there is a $d \in \mathbb{R} \backslash\{0\}$ such that $F(y)=d y$ for $y \in \mathbb{R}$. Thus, in view of (11), we get (10). Moreover $f$ is continuous on $\mathbb{R}$, so $d>0$.

Proof of Theorem 1. Since $u(0)=0$ and (4) holds for $w=0$, we have

$$
E_{g h}[u(H(X)-X)]=0 \text { for } X \in \mathcal{X}_{2} .
$$

Furthermore, as $E_{g h}$ is monotonic and $E_{g h} c=c$ for $c \in \mathbb{R}$ (cf. Lemma 1 in [4]), we get

$$
\begin{aligned}
& u(H((x, p))-x)=E_{g h}[u(H((x, p))-x)] \leq \\
& E_{g h}[u(H((x, p))-(x, p))] \leq E_{g h}[u(H((x, p)))]= \\
& u(H((x, p))) \text { for } x \in(0, \infty), p \in[0,1] .
\end{aligned}
$$

Since $u$ is strictly increasing, $u(0)=0$ and (13) holds, this implies that

$$
H((x, p))-x \leq 0 \leq H((x, p)) \text { for } x \in(0, \infty), p \in[0,1]
$$
whence

$$
H(x, p) \in[0, x] \text { for } x \in(0, \infty), p \in[0,1] .
$$

Thus, for every $x \in(0, \infty)$ and $p \in[0,1]$, the random variable

$$
\max \{u(H((x, p))-(x, p)), 0\}
$$

takes values 0 and $u(H((x, p)))$ with probabilities $p$ and $1-p$, respectively; and the random variable

$$
\max \{-u(H((x, p))-(x, p)), 0\}
$$

takes values 0 and $-u(H((x, p))-x)$ with probabilities $1-p$ and $p$, respectively. Therefore, taking into account (2) and (3), from (13) we deduce that

$$
\begin{aligned}
& g(1-p) u(H((x, p)))+h(p) u(H((x, p))-x)=0 \\
& \quad \text { for } x \in(0, \infty), p \in[0,1] .
\end{aligned}
$$

Since $u$ is strictly increasing, $u(0)=0, g(0)=h(0)=0$ and $g(1)=h(1)=1$ from (5), (14) and (15) it follows that $H((x, 0))=0$ for $x \in(0, \infty), H((x, 1))=x$ for $x \in(0, \infty)$ and

$$
H((x, p)) \in(0, x) \text { for } x \in(0, \infty), p \in(0,1) .
$$

Moreover it is not difficult to check that the continuity of $g, h$ and $u$ implies the continuity of $H((x, \cdot))$ for every $x \in(0, \infty)$ (cf. [4]). Hence

$$
\{H((x, p)) \mid p \in(0,1)\}=(0, x) \text { for } x \in(0, \infty) .
$$

Note that, in view of $(15)$, for every $x \in(0, \infty), p \in[0,1]$ and $i \in\{1,2\}$, we obtain 


$$
\begin{aligned}
& g(1-p) u\left(H\left(\left(a_{i} x, p\right)\right)\right) \\
& \quad+h(p) u\left(H\left(\left(a_{i} x, p\right)\right)-a_{i} x\right)=0 .
\end{aligned}
$$

Hence, as $H$ is $a_{i}$-scale invariant for $i \in\{1,2\}$ and every $X \in \mathcal{X}_{2}$, we get

$$
\begin{aligned}
& g(1-p) u\left(a_{i} H((x, p))\right) \\
& \quad+h(p) u\left(a_{i}(H((x, p))-x)\right)=0
\end{aligned}
$$

for $x \in(0, \infty), p \in(0,1)$ and $i \in\{1,2\}$. On the other hand, taking into account (16), from (15) we derive that

$$
\begin{aligned}
& h(p)=-\frac{u(H((x, p)))}{u(H((x, p))-x)} g(1-p) \text { for } x \in(0, \infty), \\
& p \in(0,1) .
\end{aligned}
$$

Therefore, for every $x \in(0, \infty), p \in(0,1)$ and $i \in\{1,2\}$, we have

$$
\begin{aligned}
& g(1-p)\left[u\left(a_{i} H((x, p))\right)\right. \\
& \left.\quad-\frac{u(H((x, p)))}{u(H((x, p))-x)} u\left(a_{i}(H((x, p))-x)\right)\right]=0
\end{aligned}
$$

and so, as $g(1-p)>0$ for $p \in(0,1)$, we obtain

$$
\frac{u\left(a_{i} H((x, p))\right)}{u\left(a_{i}(H((x, p))-x)\right)}=\frac{u(H((x, p)))}{u(H((x, p))-x)} .
$$

Hence, in view of (17), we get

$$
\begin{aligned}
& \frac{u\left(a_{i} y\right)}{u\left(a_{i}(y-x)\right)}=\frac{u(y)}{u(y-x)} \text { for } x \in(0, \infty), y \in(0, x), \\
& \quad i \in\{1,2\} .
\end{aligned}
$$

Setting in (20) $x=2$ and $y=1$, we obtain

$$
\frac{u\left(a_{i}\right)}{u\left(-a_{i}\right)}=\frac{u(1)}{u(-1)} \text { for } i \in\{1,2\} .
$$

Furthermore, taking a $y \in(0, \infty)$ and $x=y+1$, in view of (20), we get

$$
u\left(a_{i} y\right)=\frac{u\left(-a_{i}\right)}{u(-1)} u(y) \text { for } i \in\{1,2\} .
$$

Hence, according to (21), we have

$$
u\left(a_{i} y\right)=\frac{1}{u(1)} u\left(a_{i}\right) u(y) \text { for } y \in(0, \infty), i \in\{1,2\} .
$$

Thus, as $u$ is continuous and strictly monotonic, $\frac{1}{u(1)} u(x)>0$ for $x \in(0, \infty)$, according to Lemma, there is a $d \in(0, \infty)$ such that

$$
u(x)=u(1) x^{d} \text { for } x \in(0, \infty) .
$$

Furthermore, taking a $z \in(-\infty, 0)$, a $y \in(0, \infty)$ and applying (20) with $x=y-z$, we obtain

$$
\frac{u\left(a_{i} y\right)}{u\left(a_{i} z\right)}=\frac{u(y)}{u(z)} \text { for } i \in\{1,2\} .
$$

Hence, making use of (21) and (22), we get

$$
\tilde{u}\left(a_{i} x\right)=\frac{1}{\tilde{u}(1)} \tilde{u}\left(a_{i}\right) \tilde{u}(x) \text { for } x \in(0, \infty), i \in\{1,2\}
$$

where $\tilde{u}: \mathbb{R} \rightarrow \mathbb{R}$ is given by $\tilde{u}(x)=u(-x)$ for $x \in(0, \infty)$. Furthermore, $\frac{1}{\tilde{u}(1)} \tilde{u}(x)=\frac{1}{u(-1)} u(-x)>0$ for $x \in(0, \infty)$ and, as $u$ is continuous and strictly monotonic, so is $\tilde{u}$.
Thus, applying Lemma again, we conclude that there is a $\tilde{d} \in(0, \infty)$ such that $\tilde{u}(x)=\tilde{u}(1) x^{\tilde{d}}$ for $x \in(0, \infty)$. Therefore

$$
u(x)=u(-1)(-x)^{\tilde{d}} \text { for } x \in(-\infty, 0) .
$$

Finally, from (21)-(23) it follows that

$$
a_{i}^{d}=\frac{u\left(a_{i}\right)}{u(1)}=\frac{u\left(-a_{i}\right)}{u(-1)}=a_{i}^{\tilde{d}} \text { for } i \in\{1,2\},
$$

which implies that $\tilde{d}=d$. So, taking into account $(22)$ and (23), we obtain (6) with $\alpha:=u(1)>0$ and $\beta:=$ $-u(-1)>0$.

A straightforward calculation shows that if (2) holds and $u$ is of the form (6) with some $\alpha, \beta, d>0$, then $H$ is scale invariant.

Proof of Theorem 2. According to Theorem 1, $u$ is of the form (6) with some $\alpha, \beta, d>0$. Moreover, arguing as in the proof of Theorem 1 , we obtain that (17) and (19) hold and, for every $x \in(0, \infty), H((x, \cdot))$ is continuous and $H((x, 1))=x$. Thus, taking $\varepsilon:=\min \left\{w_{0}, \frac{w_{0}}{a_{1}}, \frac{w_{0}}{a_{2}}\right\}$, we conclude that for every $x \in(\varepsilon, \infty)$ there exists a $p_{x} \in$ $(0,1)$ such that

$$
\left\{H((x, p)) \mid p \in\left(p_{x}, 1\right)\right\}=(x-\varepsilon, x) .
$$

Furthermore, for every $x \in(\varepsilon, \infty), p \in\left(p_{x}, 1\right)$ and $i \in$ $\{1,2\}$, we have

$$
w_{0}+H((x, p))-x>w_{0}-\varepsilon \geq 0
$$

and

$$
w_{0}+a_{i}(H((x, p))-x)>w_{0}-a_{i} \varepsilon \geq 0 .
$$

Therefore, as $H$ is $a_{i}$-scale invariant for $i \in\{1,2\}$ and every $X \in \mathcal{X}_{2}$, taking into account (2), (3), (4) (with $w=$ $\left.w_{0}\right)$ and $(6)$, for every $x \in(\varepsilon, \infty), p \in\left(p_{x}, 1\right)$ and $i \in$ $\{1,2\}$, we obtain

$$
\begin{aligned}
& w_{0}^{d}=(1-g(1-p))\left(w_{0}+H((x, p))-x\right)^{d} \\
& \quad+g(1-p)\left(w_{0}+H((x, p))\right)^{d}
\end{aligned}
$$

and

$$
\begin{aligned}
& w_{0}^{d}=(1-g(1-p))\left(w_{0}+a_{i}(H((x, p))-x)\right)^{d} \\
& \quad+g(1-p)\left(w_{0}+a_{i} H((x, p))\right)^{d} .
\end{aligned}
$$

From (25) it follows that

$$
g(1-p)=\frac{w_{0}^{d}-\left(w_{0}+H((x, p))-x\right)^{d}}{\left(w_{0}+H((x, p))\right)^{d}-\left(w_{0}+H((x, p))-x\right)^{d}}
$$

for $x \in(\varepsilon, \infty)$ and $p \in\left(p_{x}, 1\right)$ which, together with (26), gives

$$
\begin{aligned}
& \frac{w_{0}^{d}-\left(w_{0}+a_{i}(H((x, p))-x)\right)^{d}}{w_{0}^{d}-\left(w_{0}+H((x, p))-x\right)^{d}}= \\
& \quad \frac{\left(w_{0}+a_{i} H((x, p))\right)^{d}-\left(w_{0}+a_{i}(H((x, p))-x)\right)^{d}}{\left(w_{0}+H((x, p))\right)^{d}-\left(w_{0}+H((x, p))-x\right)^{d}}
\end{aligned}
$$

for $x \in(\varepsilon, \infty), p \in\left(p_{x}, 1\right)$ and $i \in\{1,2\}$. Thus

$$
\begin{aligned}
& \frac{v\left(a_{i}(H((x, p))-x)\right)}{v(H((x, p))-x)}= \\
& \frac{v\left(a_{i} H((x, p))\right)-v\left(a_{i}(H((x, p))-x)\right)}{v(H((x, p)))-v(H((x, p))-x)}
\end{aligned}
$$


for $x \in(\varepsilon, \infty), p \in\left(p_{x}, 1\right)$ and $i \in\{1,2\}$, where $v: \mathbb{R} \rightarrow \mathbb{R}$ is given by

$$
v(x)=\left(w_{0}+x\right)^{d}-w_{0}^{d} \text { for } x \in \mathbb{R} .
$$

Hence, in view of (24), we get

$$
\frac{v\left(a_{i}(y-x)\right)}{v(y-x)}=\frac{v\left(a_{i} y\right)-v\left(a_{i}(y-x)\right)}{v(y)-v(y-x)} .
$$

for $x \in(\varepsilon, \infty), y \in(x-\varepsilon, x), i \in\{1,2\}$. So, after a standard calculation, we conclude that

$$
\begin{aligned}
& \frac{v\left(a_{i}(y-x)\right)}{v(y-x)}=\frac{v\left(a_{i} y\right)}{v(y)} \text { for } x \in(\varepsilon, \infty), \\
& \quad y \in(x-\varepsilon, x), i \in\{1,2\} .
\end{aligned}
$$

Let $n \in \mathbb{N}$ be such that $n>\max \{1, \varepsilon\}$. Then, applying (28) with $x=y+\left(1-\frac{1}{n}\right) \varepsilon$, where $y \in\left(\frac{\varepsilon}{n}, \infty\right)$, we obtain

$$
\begin{aligned}
& v\left(a_{i} y\right)=\frac{v\left(-a_{i}\left(1-\frac{1}{n}\right) \varepsilon\right)}{v\left(-\left(1-\frac{1}{n}\right) \varepsilon\right)} v(y) \text { for } y \in\left(\frac{\varepsilon}{n}, \infty\right), \\
& i \in\{1,2\} .
\end{aligned}
$$

Taking in this equality $y=1 \in\left(\frac{\varepsilon}{n}, \infty\right)$, we get

$$
\frac{v\left(a_{i}\right)}{v(1)}=\frac{v\left(-a_{i}\left(1-\frac{1}{n}\right) \varepsilon\right)}{v\left(-\left(1-\frac{1}{n}\right) \varepsilon\right)} \text { for } i \in\{1,2\} \text {. }
$$

From the last two equalities we derive that

$$
v\left(a_{i} y\right)=\frac{1}{v(1)} v\left(a_{i}\right) v(y) \text { for } y \in\left(\frac{\varepsilon}{n}, \infty\right), i \in\{1,2\}
$$

and so, as $n \in \mathbb{N}$ with $n>\max \{1, \varepsilon\}$ is fixed arbitrarily, $\mathrm{w}$ get

$$
v\left(a_{i} y\right)=\frac{1}{v(1)} v\left(a_{i}\right) v(y) \text { for } y \in(0, \infty), i \in\{1,2\}
$$

Since $v$ is continuous and strictly monotonic, $\frac{1}{v(1)} v(y)>0$ for $y \in(0, \infty)$, applying Lemma, we conclude that there is an $r \in(0, \infty)$ such that $v(y)=v(1) y^{r}$ for $y \in(0, \infty)$. Hence, in view of (27), we get

$$
\left(w_{0}+y\right)^{d}-w_{0}^{d}=v(1) y^{r} \text { for } y \in(0, \infty) .
$$

Therefore

$$
\begin{aligned}
0< & d w_{0}^{d-1}=\left(y^{d}\right)_{y=w_{0}}^{\prime}=\lim _{y \rightarrow 0^{+}} \frac{\left(w_{0}+y\right)^{d}-w_{0}^{d}}{y}= \\
& \lim _{y \rightarrow 0^{+}} v(1) y^{r-1} .
\end{aligned}
$$

This implies that $r=1$, which together with (29), gives

$$
\left(w_{0}+y\right)^{d}=v(1) y+w_{0}^{d} \text { for } y \in(0, \infty) .
$$

Differentiating both sides of this equality with respect to $y$, we obtain

$$
d\left(w_{0}+y\right)^{d-1}=v(1) \text { for } y \in(0, \infty) .
$$

Hence $d=1$ and so, making use of (6), we conclude that

$$
u(x)= \begin{cases}\alpha x & \text { for } x \geq 0, \\ \beta x & \text { for } x<0 .\end{cases}
$$

Fix a $p \in(0,1)$ and suppose that a function

$$
(0, \infty) \ni x \rightarrow H((x, p))-x
$$

is bounded below, say $H((x, p))-x \geq m$ for $x \in(0, \infty)$ with some $m \in \mathbb{R}$. Then $\lim _{x \rightarrow \infty} H((x, p))=\infty$ and so, in view of $(30)$, we get

$$
\begin{aligned}
& \lim _{x \rightarrow \infty} g(1-p) u\left(a_{1} H((x, p))\right)= \\
& \quad \lim _{x \rightarrow \infty} g(1-p) \alpha a_{1} H((x, p))=\infty .
\end{aligned}
$$

On the other hand, as $u$ is strictly increasing, we get

$$
\begin{aligned}
& h(p) u\left(a_{1}(H((x, p))-x)\right) \geq h(p) u\left(a_{1} m\right) \\
& \quad \text { for } x \in(0, \infty) .
\end{aligned}
$$

This contradicts (19). Thus the function given by (31) is unbounded below. Hence there is an $x \in(0, \infty)$ such that $H((x, p))-x<-\frac{w_{0}}{a_{i}}$ for $i \in\{1,2\}$. Therefore, as $H$ is $a_{i^{-}}$ scale invariant for $i \in\{1,2\}$ and every $X \in \mathcal{X}_{2}$, applying (2), (3), (4) (with $w=w_{0}$ ) and (30), for $i \in\{1,2\}$, we obtain

$$
\begin{gathered}
\alpha w_{0}=g(1-p) \alpha\left(w_{0}+a_{i} H((x, p))\right) \\
+h(p) \beta\left(w_{0}+a_{i}(H((x, p))-x)\right) .
\end{gathered}
$$

Hence

$$
\begin{aligned}
& {[(g(1-p) \alpha+h(p) \beta) H((x, p))-h(p) \beta x] a_{i}=} \\
& \quad[(1-g(1-p)) \alpha-h(p) \beta] w_{0}
\end{aligned}
$$

for $i \in\{1,2\}$. Thus

$$
[(g(1-p) \alpha+h(p) \beta) H((x, p))-h(p) \beta x]\left(a_{1}-a_{2}\right)=0
$$

and so, as $a_{1} \neq a_{2}$, we get

$$
(g(1-p) \alpha+h(p) \beta) H((x, p))-h(p) \beta x=0 .
$$

Therefore from $(32)$ we derive that $(1-g(1-p)) \alpha-$ $h(p) \beta=0$. Since $p \in(0,1)$ is fixed arbitrarily, this means that

$$
(1-g(1-p)) \alpha-h(p) \beta=0 \text { for } p \in(0,1) .
$$

As $g(0)=0$ and $h(1)=1$, letting in $(33) p \rightarrow 1^{-}$, we obtain that $\alpha=\beta$. Thus, in view of (30) and (33), we get (8) and (7), respectively.

\section{Conclusion}

The Principle of Equivalent Utility is one of the most frequently applied methods of pricing insurance contracts. Recently, Kałuszka and Krzeszowiec [4] introduced a modification of the Principle of Equivalent Utility adjusted to the Cumulative Prospect Theory and studied several its properties. One of them is a scale invariance. We have proved that a scale invariance of the Principle of Equivalent Utility just for two values of the scaling parameters implies its scale invariance for all parameters. In particular, we generalized the result from the paper of Reich [6] where, among others, the similar result has been proved under the Expected Utility Theory.

\section{References}

[1] J. Quiggin, J. Econ. Behav. Organ. 3, 323 (1982).

[2] U. Segal, Ann. Oper. Res. 19, 359 (1989).

[3] A. Tversky, D. Kahneman, J. Risk Uncertainty 5, 297 (1992).

[4] M. Kałuszka, M. Krzeszowiec, Insur. Math. Econ. 50, 159 (2012).

[5] S. Heilpern, Insur. Math. Econ. 33, 67 (2003).

[6] A. Reich, ASTIN Bulletin 14, 123 (1984).

[7] M. Kuczma, An Introduction to the Theory of Functional Equations and Inequalities, Birkhäuser, Berlin 2009 . 